\title{
Aplicação de Inteligência Artificial na Avaliação de Confiabilidade Composta de Sistemas de Potência
}

\author{
Daniel T. Kitamura* Karolayne P. M. Faria** \\ Danrlei D. Oliveira*** Leonardo W. Oliveira**** Guilherme H. Gonçalves $\dagger$ Edimar José de Oliveirax
}

\author{
*Faculdade de Engenharia, Universidade Federal de Juiz de Fora, MG, Brasil \\ (Tel: 5532 99920-3403; e-mail: daniel.kitamura@engenharia.ufjf.br). \\ **Faculdade de Engenharia, Universidade Federal de Juiz de Fora, MG, Brasil \\ (e-mail:karolayne.martins@engenharia.ufjf.br). \\ ***Faculdade de Engenharia, Universidade Federal de Juiz de Fora, MG, Brasil \\ (e-mail: danrlei.diegues@engenharia.ufjf.br). \\ ****Faculdade de Engenharia, Universidade Federal de Juiz de Fora, MG, Brasil \\ (e-mail:leonardo.willer@engenharia.ufj..br). \\ $\dagger$ Faculdade de Engenharia, Universidade Federal de Juiz de Fora, MG, Brasil \\ (e-mail: guilherme.goncalves@engenharia.ufj.br). \\ $\times$ Faculdade de Engenharia, Universidade Federal de Juiz de Fora, MG, Brasil \\ (e-mail: edimar.oliveira@ufjf.edu.br).
}

\begin{abstract}
The present paper proposes an analysis of composite reliability of power system with aid of the artificial intelligence technique known as Artificial Neural Network (ANN). The model for evaluating reliability is based on the method of Non-Sequential Monte Carlo Simulation (NS-MCS) with redispatch of generation through an Optimal Power Flow (OPF). The ANN is applied to determine condition of load shedding and to avoid the OPF in case of no load shedding. As a contribution, the paper proposes the analysis of different inputs of the ANN, as the total available generation and the available generation by bus. In order to assess the proposed approach, the IEEE-14 and IEEE-118 test systems are used, where the impacts of the ANN input variables can be evaluated.
\end{abstract}

Resumo: O presente artigo propõe a análise de confiabilidade composta de sistemas de potência com suporte da técnica de inteligência artificial Redes Neurais Artificiais (RNA). O modelo de avaliação de confiabilidade baseia-se no método de Simulação de Monte Carlo Não Sequencial (SMC-NS) com redespacho de geração via Fluxo de Potência Ótimo (FPO). A RNA é aplicada para determinar condição de corte de carga e evitar a execução do FPO para redespacho em caso de ausência de corte. Como contribuição na análise, o artigo propõe a análise de diferentes entradas para a RNA, como geração total disponível e a geração disponível por barra. Para avaliar a metodologia proposta, os sistemas testes IEEE14 e IEEE-118 são utilizados, em que se pode verificar o impacto das variáveis de entrada da RNA.

Keywords: composite reliability; artificial intelligence; artificial neural networks; power systems; Monte Carlo Simulation.

Palavras-chaves: confiabilidade composta; inteligência artificial; redes neurais artificiais; sistemas de potência; Simulação Monte Carlo.

\section{INTRODUÇÃO}

Os Sistemas Elétricos de Potência (SEP) têm a função de fornecer energia elétrica aos seus consumidores de forma econômica, segura e confiável. Deve-se, portanto, encontrar o ponto ótimo entre estes três fatores. Neste esforço, estudos de confiabilidade são importantes para o planejamento da expansão de SEP. Tais estudos avaliam a capacidade do sistema de cumprir sua função dentro de limites operativos, considerando possíveis falhas de equipamentos. São considerados três níveis hierárquicos para estes estudos: NH1, NH2 e NH3. O nível NH1 considera apenas o sistema de geração, o NH2 incorpora as restrições de transmissão e, por fim, o NH3 inclui a distribuição. O nível hierárquico NH2 resulta na análise conhecida como confiabilidade composta, que pode ser realizada por duas classes de métodos: determinísticos ou probabilísticos. Nos métodos determinísticos, a partir do conhecimento prévio do sistema, faz-se a avaliação de condições operativas incluindo contingências, cujos critérios mais comuns são o "N-1" e o "N2", em que o sistema apresenta contingências simples e duplas, respectivamente. A limitação destes métodos é não representar a natureza estocástica de SEP, podendo gerar a resultados muito conservadores ou pouco significativos (Allan e Billinton 2000).

Os métodos probabilísticos representam a natureza estocástica de SEP a partir da probabilidade de ocorrência de determinada condição operativa. Tais métodos são mais complexos e necessitam de dados estocásticos, porém, são capazes de encontrar resultados mais significativos. Dentre os métodos 
probabilísticos, pode-se citar a representação por espaço de estados, como a enumeração de estados, computacionalmente mais dispendiosa, e os baseados em Simulação de Monte Carlo (SMC) (Billinton e Li 1994), (Pereira e Balu 1992), (Mello et al. 1994), (Leite da Silva et al. 2000). Na SMC não sequencial (SMC-NS), os estados do sistema são amostrados aleatoriamente sem qualquer representação cronológica, enquanto que na SMC sequencial (SMC-S), os estados são amostrados sequencialmente durante um período de tempo, porém, com maior esforço computacional. Alguns métodos propõem estratégias para redução do esforço computacional da SMC-S, como a SMC pseudo-sequencial (Mello et al. 1994) e a SMC pseudo-cronológica (Leite da Silva et al. 2000).

Técnicas de inteligência artificial (IA) também têm sido utilizadas para reduzir o esforço computacional da SMC aplicada à análise de confiabilidade composta. Em Mitra e Xu (2010), a otimização por enxame de partículas é proposta para identificar estados em que ocorrem corte de carga. A mesma ideia foi utilizada em Xu e Mitra (2011), porém, utilizando o algoritmo inspirado em cultura de bactérias. Em Luo et al. (2000), uma Rede Neural Artificial (RNA) do tipo mapa autoorganizável, do inglês Self Organizing Map (SOM), é aplicada para aumentar a eficiência da SMC-NC visando estimar o índice de probabilidade de perda de carga. Em Song et al. (2005), a RNA do tipo SOM combinada com fuzzy, Fuzzy Self Organizing Map (FSOM), é utilizada juntamente com a SMC$\mathrm{S}$ para identificar estados falhos. Em (Leite da Silva et al. 2007), propõe-se a utilização de RNA combinada com a SMCNS para a avaliação de confiabilidade do sistema por áreas ou barras. Em Resende et al. (2015), propõe-se a técnica baseada em máquina de vetor de suporte combinada com a SMC-NS para avaliar a confiabilidade em todos níveis (sistema, áreas e barras). Recentemente, a mesma ideia foi proposta em Urgun et al. (2018).

Para avaliar os índices de confiabilidade, a SMC pode ser dividida em três estágios: seleção dos estados, avaliação dos mesmos e cálculo dos índices (Rosa et al. 2012). A RNA utilizada no presente trabalho é aplicada ao segundo estágio para reconhecer estados falhos em que há corte de carga e, consequentemente, estados não falhos, evitando, assim, a análise de fluxo de potência em casos de ausência de corte (sucesso). No presente artigo, a RNA denominada Perceptron Multicamadas, do inglês Multilayer Perceptron, é combinada com a SMC-NS para avaliar os índices de probabilidade de perda de carga e de energia esperada não suprida. A contribuição é avaliar o efeito de entradas na RNA, como a geração total disponível e a geração disponível por barra. A metodologia via RNA é comparada com a SMC-NS tradicional, sem a aplicação de RNA, utilizando-se dados de dois sistemas testes da literatura: IEEE-14 e IEEE-118.

\section{MODELO DE AVALIAÇÃO DE CONFIABILIDADE COMPOSTA}

\subsection{Simulação de Monte Carlo Não Sequencial}

A SMC-NS (Billinton e Li 1993) baseia-se na amostragem aleatória e em larga escala de estados de um sistema, sem considerar a ordem cronológica de seus eventos. Assim, a seleção dos estados é feita através de distribuições de probabilidades e das relações de seus componentes. Desta maneira, pode-se determinar o estado de um equipamento através de sua taxa de saída forçada, do inglês Forced Outage Rate (FOR), e de uma amostra aleatória de probabilidades dentro de uma distribuição uniforme. Caso a probabilidade amostrada para um equipamento seja inferior à sua FOR, o estado é de falha e caso contrário, o estado é operante. A partir dos estados de cada componente de rede, é possível determinar o estado do SEP.

Para a análise de desempenho de cada um desses estados, funções teste $(F t)$ são utilizadas, cujos valores esperados são formulados como:

$$
E[F t]=\sum_{x \in X} F t(x) * P(x)
$$

Em que $P(x)$ representa a probabilidade do estado " $x$ " $\mathrm{e}$ " $X$ ", o conjunto de todos os estados do sistema. Na SMC, obtém-se uma estimativa $(\tilde{E})$ para o valor esperado da função teste. Como a técnica em questão realiza a amostragem de "NS" estados, os índices são estimados pela média das funções $F t$, ou seja:

$$
\tilde{\mathrm{E}}(F t)=\frac{1}{N S} \sum_{k=1}^{N S} F t\left(X^{k}\right)
$$

A variância da estimativa da função $F t, \tilde{V}(\tilde{E}(F t))$, é dada em função da variância de $F t$, como:

$$
\tilde{\mathrm{V}}(\tilde{\mathrm{E}}(F t))=\frac{\tilde{\mathrm{V}}(F t)}{N S}
$$

Após a determinação da estimativa e da variância da estimativa do valor esperado de $F t(x)$, calcula-se o coeficiente de variação $\beta$ formulado em (4).

$$
\beta=\frac{\sqrt{\tilde{\mathrm{V}}(\tilde{\mathrm{E}}(F t))}}{\tilde{\mathrm{E}}(F t)} * 100 \%
$$

O coeficiente de variação $\beta$ é a tolerância para o cálculo dos índices. Ou seja, é um dos critérios de parada para a SMC-NS. Observa-se que a variância, que representa a dispersão dos valores esperados $(\tilde{\mathrm{E}}(F t))$ da função teste $(F t)$, é utilizada para o cálculo do coeficiente $\beta$. Outro critério de convergência é o número máximo de sorteios (Nmáx).

No presente trabalho, as funções teste utilizadas são:

- $\quad$ Probabilidade de perda de carga (LOLP);

- $\quad$ Expectativa de perda de carga (LOLE) (horas);

- $\quad$ Potência esperada não suprida (EPNS) (MW);

- $\quad$ Energia esperada não suprida (EENS) (MWh).

Os índices anteriores são formulados como (Dias 2014):

$$
L O L P=\sum_{i \in S} p_{i}
$$




$$
\begin{aligned}
L O L E & =L O L P * T \\
E P N S & =\sum_{i \in S} C_{i} * p_{i} \\
\text { EENS } & =\text { EPNS } * T
\end{aligned}
$$

(8) Em que, $\alpha_{k}$ representa os corte de carga na barra " $k$ "; Ic o

sorteios (Nmáx), utilizados como critérios de convergência do algoritmo;

2) Inicializar a variável referente ao número de sorteios como zero $(N S=0)$;

3) Sortear um estado do sistema $x \in X$ que irá corresponder a um ponto de operação, (demanda de carga e geração) e incrementar a variável "NS";

4) Realizar o cálculo da função teste Ft(x) como sendo a média da função de saída obtida até então (valor de "Ft" para cada estado " $x$ " do sistema)

5) Realizar o cálculo da estimativa da função teste, $\tilde{\mathrm{E}}(F t)$;

6) Analisar o critério de convergência: $\beta$ menor que uma tolerância pré-estabelecida elou número máximo de sorteios alcançado;

7) Caso algum dos critérios de convergência seja atendido, o algoritmo termina; caso contrário, retorna ao passo 3 ).

\subsection{Fluxo de Potência Ótimo (FPO)}

No presente trabalho, um modelo de fluxo de potência ótimo é utilizado para identificar se um estado é ou não falho (Terra 1989), (Oliveira e Filho 2003), a fim de gerar dados de treinamento para a RNA. Este FPO considera o modelo linearizado (DC) da rede elétrica, aceitável para análise de fluxo de carga de sistemas de transmissão no contexto da confiabilidade composta. O objetivo do modelo de FPO utilizado é minimizar o corte de carga no sistema, quando necessário, no passo 4 do algoritmo descrito na subseção anterior, através de redespacho de unidades geradoras. Portanto, este modelo pode ser formulado como (Carvalho et al. 1988):

$$
\begin{gathered}
\min \sum_{k \in I c} \alpha_{k} * P D_{k} \\
\text { s.a: } \sum_{j \in \Omega k} P_{k j}=P G_{k}-P D_{k} *\left(1-\alpha_{k}\right)
\end{gathered}
$$
conjunto de barras de carga; $f_{k j}$ a potência ativa na linha $k-j$; $P G_{k}$ e $P D_{k}$ as potências gerada e de carga na barra " $k$ ", respectivamente; $\Omega k$ o conjunto de barras conectadas à barra " $k$ " através de linhas de transmissão, $\overline{P G}_{k}$ e $\bar{f}_{k j}$ são os limites de $P G_{k}$ e $f_{k j}$, respectivamente.

A restrição (10) representa o balanço de potência em cada barra conforme dada pela segunda lei de Kirchoff. As restrições (11) e (12), por sua vez, estabelecem limites para as variáveis de fluxo em linhas e geração, respectivamente.

\section{METODOLOGIA PROPOSTA}

$$
\begin{gathered}
-\bar{f}_{k j} \leq f_{k j} \leq \bar{f}_{k j} \\
-\overline{P G}_{k} \leq P G_{k} \leq \overline{P G}_{k}
\end{gathered}
$$

A metodologia aplicada no presente trabalho consiste em incluir a RNA, também capaz de identificar estados falhos, porém, com um tempo de identificação muito menor do que o necessário para a execução do FPO, no passo 4 do algoritmo da subseção 2.1, como suporte a ele. A capacidade da RNA de distinguir estados de sucesso e de falha baseia-se no fato de que estas categorias de estado apresentam padrões típicos. A partir disso, a rede é capaz de identificar estes padrões e classificar amostras em cada padrão (Resende et al. 2015).

A RNA Multilayer Perceptron apresenta uma camada de entrada que recebe o conjunto de dados, uma camada oculta que recebe estas informações e as processa, e uma camada de saída que apresenta a resposta da rede. A saída da RNA utilizada é binária, em que o valor " 0 " representa estado de sucesso e o valor "1" representa estado de falha. As Fig. 1 e 2 ilustram a arquitetura da RNA utilizada nas duas opções avaliadas neste trabalho, descritas a seguir:

- Opção-1: Uma entrada, sendo a capacidade total de geração de potência disponível $(\overline{P G e})$ em cada estado " $e$ ";

- Opção-2: As capacidades de geração disponível por barras no estado " $e$ " $\left(\overline{P G e}_{k}\right)$.

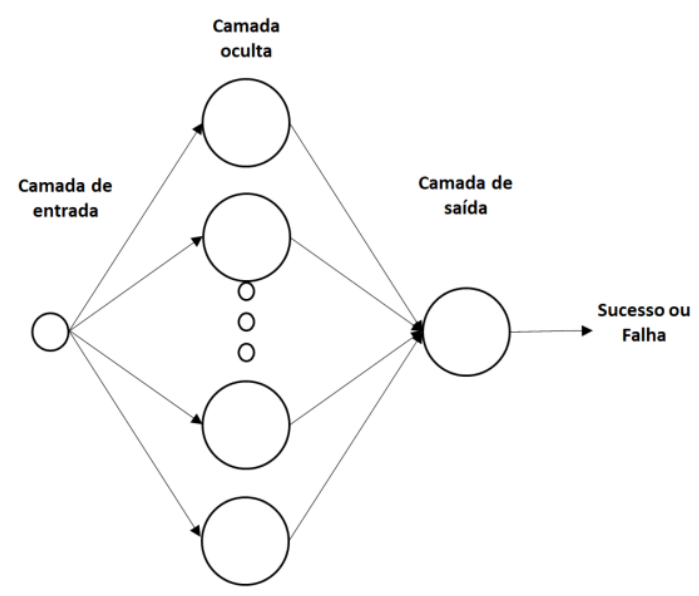

Fig. 1 Estrutura da RNA utilizada na Opção-1. 


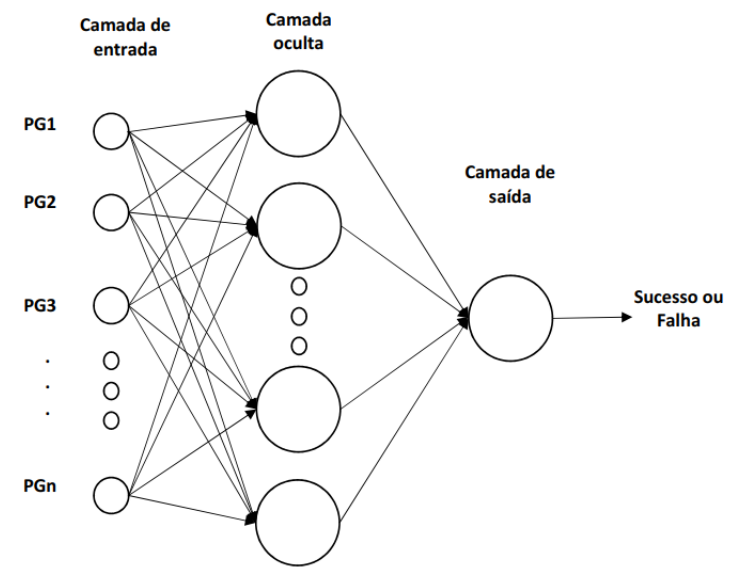

Fig. 2 Estrutura da RNA utilizada na Opção-2.

Destaca-se que a reserva de geração e a capacidade indisponível por área são utilizadas como entradas em Leite da Silva et al. (2007). Em Resende et al. (2015), a capacidade de geração disponível e a indisponibilidade dos componentes da geração são utilizadas. Já em Urgun e Singh (2018), a capacidade total de geração por barra também é usada.

Para o processo de treinamento da RNA, um conjunto de estados é selecionado dentro do espaço amostral gerado pela SMC-NS (capacidade de geração disponível). A análise de falha é feita utilizando-se o FPO, a fim de prover os padrões de saída esperados para RNA (sucesso ou falha). O algoritmo de treinamento utilizado foi por retropropagação elástica, do inglês backpropagation. O critério de convergência é dado pelo erro médio quadrático formulado a seguir:

$$
\in=\left[\frac{1}{N} \sum_{i=1}^{N}\left(Y_{i}-Y_{i}^{*}\right)^{2}\right]^{1 / 2}
$$

Em que, $N$ é o número de pares entrada-saída da RNA, $Y_{i}$ é o valor obtido da RNA para a variável de saída $i$, e $Y_{i}^{*}$ é o valor esperado da RNA para a variável de saída $i$.

Após treinada, a RNA dá suporte ao FPO do passo 4 do algoritmo da subseção 2.1, visando reduzir o tempo de execução da SMC-NS. Nesta aplicação, as mesmas variáveis utilizadas para treinamento da RNA, conforme Opção-1 ou 2, são submetidas à entrada da rede, que identifica os estados. Caso um estado seja identificado como de falha, o FPO descrito na subseção 2.2 é executado para determinar o mínimo corte de carga necessário. Por outro lado, se o estado identificado pela RNA for de sucesso, então o FPO não é executado, uma vez que, neste caso, não há corte de carga. Portanto, a não execução do FPO em casos de sucesso permite reduzir consideravelmente o tempo de execução da SMC-NS.

A metodologia utilizada pode ser descrita em quatro passos, conforme o algoritmo a seguir:

1) Obtenha um estado amostrado " $s$ " por meio da SMC-NS;

2) Com a RNA já treinada, classifique o estado " $s$ " como sucesso ou falha através da RNA, com base nas amostradas geradas por meio da SMC-NS associada ao FPO. Caso haja falha, vá ao próximo passo, caso contrário, vá ao passo 4;

3) Execute o FPO para determinar o mínimo corte de carga do sistema para o estado " $s$ ";

4) Estime os índices de confiabilidade. Se um dos critérios de convergência for alcançado, então pare. Caso contrário, retorne ao passo 1.

\section{ESTUDO DE CASOS}

A fim de realizar análises envolvendo a aplicação de RNA ao problema de avaliação de confiabilidade composta de SEP, considerando diferentes opções de entrada, dois sistemas do IEEE são utilizados: IEEE-14 e IEEE-118 (Electrical and Computer Engineering 2019). Para ambos, a RNA utilizada no presente trabalho apresenta número de entradas conforme Opções- 1 e 2 anteriormente definidas, uma camada oculta com 30 neurônios e uma saída binária. A tolerância para a convergência da $\operatorname{SMC}(\beta)$ é de $1 * 10^{-4}$ para o LOLP e de $5^{*} 10^{-}$ ${ }^{3}$ para o EPNS. O número máximo de sorteios (Nmáx) é igual a 10000. A tolerância do erro médio quadrático do conjunto de treinamento para convergência da RNA é de $1 * 10^{-4}$.

Nos dois sistemas analisados, três casos de análise são considerados.

- Caso-1: SMC-NS tradicional, ou seja, sem a utilização de RNA;

- Caso-2: SMC-NS com RNA na Opção-1 (Uma entrada, sendo a capacidade total de geração de potência disponível em cada estado;

- Caso-3: SMC-NS com RNA na Opção-2 (Entradas: capacidades de geração disponível por barras, em cada estado).

As simulações de todos os Casos foram realizadas no software MATLAB, em um processador Intel Core i7 6500U CPU @ 2,50 GHz 2,59 GHz, 8,00 GB RAM.

\subsection{Sistema IEEE-14}

Para o sistema IEEE-14, os parâmetros para avaliação de confiabilidade considerados são apresentados nas Tabelas $1 \mathrm{e}$ 2. Um total de 10000 amostras de pares geração/corte de carga é utilizado, sendo dividido em conjuntos de treinamento (5000 amostras), validação (2500 amostras) e teste (2500 amostras). Este número mostrou-se satisfatório para os estudos de caso realizados.

Tabela 1. Parâmetros de confiabilidade de geração, IEEE-14.

\begin{tabular}{|c|c|c|c|c|c|}
\hline $\begin{array}{c}\text { Barra do } \\
\text { Gerador }\end{array}$ & $\begin{array}{c}\text { Barra } \\
\text { "1" }\end{array}$ & $\begin{array}{c}\text { Barra } \\
\text { "2" }\end{array}$ & $\begin{array}{c}\text { Barra } \\
\text { "3" }\end{array}$ & $\begin{array}{c}\text { Barra } \\
\text { "6" }\end{array}$ & $\begin{array}{c}\text { Barra } \\
\text { "8" }\end{array}$ \\
\hline $\begin{array}{c}\text { Taxa de Saída } \\
\text { Forçada (\%) }\end{array}$ & 4 & 4 & 1 & 1 & 4 \\
\hline
\end{tabular}


Tabela 2. Parâmetro de confiabilidade de linhas, IEEE-14.
Taxa de Saída Forçada (\%)
0,0223

A Tabela 3 apresenta os resultados obtidos para os índices de confiabilidade em cada Caso anteriormente definido. Os desvios percentuais apresentados para os Casos-2 e 3 referemse aos erros percentuais dos respectivos valores obtidos, em relação aos valores do Caso-1 para a SMC-NS convencional sem aplicação de RNA. A Tabela 4 apresenta os erros médios quadráticos da RNA para os conjuntos de treinamento, validação e teste.

Tabela 3. Resultados, IEEE-14.

\begin{tabular}{|c|c|c|c|}
\hline Índice & Caso-1 & Caso-2 & Caso-3 \\
\hline LOLP & 0,0099 & $\begin{array}{c}0,0119 \\
(\text { desvio } \\
20 \%)\end{array}$ & $\begin{array}{c}0,0078 \\
(\text { desvio } \\
21 \%)\end{array}$ \\
\hline $\begin{array}{c}\text { LOLE } \\
\text { (horas) }\end{array}$ & 86,9478 & $\begin{array}{c}104,45 \\
(\text { desvio } \\
20 \%)\end{array}$ & $\begin{array}{c}68,55 \\
(\text { desvio } \\
21 \%)\end{array}$ \\
\hline $\begin{array}{c}\text { EPNS } \\
\text { (MW) }\end{array}$ & 0,3299 & $\begin{array}{c}0,4280 \\
(\text { desvio } \\
30 \%)\end{array}$ & $\begin{array}{c}0,2837 \\
(\text { desvio } \\
14 \%)\end{array}$ \\
\hline $\begin{array}{c}\text { EENS } \\
\text { (MWh) }\end{array}$ & $2.890,20$ & $\begin{array}{c}3.749,6 \\
(\text { desvio } \\
30 \%)\end{array}$ & $\begin{array}{c}2.485,1 \\
(\text { desvio } \\
14 \%)\end{array}$ \\
\hline $\begin{array}{c}\text { Tempo } \\
\text { (min) }\end{array}$ & 3,52 & 2,51 & 2,47 \\
\hline
\end{tabular}

Tabela 4. Erros médios quadráticos da RNA, IEEE-14.

\begin{tabular}{|c|c|c|c|}
\hline Caso & $\begin{array}{c}\text { Conjunto de } \\
\text { Treinamento }\end{array}$ & $\begin{array}{c}\text { Conjunto de } \\
\text { Validação }\end{array}$ & $\begin{array}{c}\text { Conjunto de } \\
\text { Teste }\end{array}$ \\
\hline Caso-2 & 0,0012 & 1,4050 & 1,5767 \\
\hline Caso-3 & 0,0015 & 1,4262 & 1,8880 \\
\hline
\end{tabular}

Da Tabela 3, pode-se observar que o Caso-3, associado à segunda opção de RNA (gerações disponíveis por barra) resulta no menor desvio percentual de EPNS e EENS em relação ao resultado da SMC-NS convencional (Caso-1). Com relação a LOLP e LOLE, obtém praticamente o mesmo desempenho do Caso-2 (geração disponível total). Observa-se ainda o ganho de eficiência computacional, através da redução do tempo de execução com a aplicação das RNAs, ou seja, deve-se avaliar se a perda de precisão justifica o ganho de eficiência computacional em análises requeridas por gerenciadores de sistemas de energia. No entanto, a análise realizada no presente trabalho já provê condições para a avaliação de custo-benefício entre estes critérios (precisão e tempo), embora, para sistemas de maior porte, espera-se que este impacto seja maior.

\subsection{Sistema IEEE-118}

Para o sistema IEEE-118, assim como no IEEE-14, 10000 amostras de pares geração / corte de carga são utilizadas, divididas entre conjunto de treinamento com 5000 amostras e conjuntos de validação e teste com 2500 amostras cada. O parâmetro de confiabilidade de linhas são os mesmos do sistema IEEE-14.

Os resultados obtidos para o sistema de IEEE-118, nos três Casos anteriormente definidos, são apresentados na Tabela 5. A Tabela 6 apresenta os erros médios quadráticos obtidos para este sistema.

Tabela 5. Resultados, IEEE-118.

\begin{tabular}{|c|c|c|c|}
\hline Índice & Caso-1 & Caso-2 & Caso-3 \\
\hline LOLP & 0,0696 & $\begin{array}{c}0,0684 \\
(\text { desvio } 2 \%)\end{array}$ & $\begin{array}{c}0,0726 \\
(\text { desvio } 4 \%)\end{array}$ \\
\hline $\begin{array}{c}\text { LOLE } \\
\text { (horas })\end{array}$ & 609,84 & $\begin{array}{c}599,43 \\
(\text { desvio } 2 \%)\end{array}$ & $\begin{array}{c}636,29 \\
(\text { desvio } 4 \%)\end{array}$ \\
\hline $\begin{array}{c}\text { EPNS } \\
\text { (MW) }\end{array}$ & 1,75 & $\begin{array}{c}1,78 \\
(\text { desvio } 2 \%)\end{array}$ & $\begin{array}{c}1,81 \\
(\text { desvio 3\%) }\end{array}$ \\
\hline $\begin{array}{c}\text { EENS } \\
\text { (MWh) }\end{array}$ & $15.326,00$ & $\begin{array}{c}15.573,6 \\
(\text { desvio } 2 \%)\end{array}$ & $\begin{array}{c}15.842,00 \\
(\text { desvio 3\%) }\end{array}$ \\
\hline $\begin{array}{c}\text { Tempo } \\
(\mathrm{min})\end{array}$ & 17,5 & 12,24 & 12,26 \\
\hline
\end{tabular}

Tabela 6. Erros médios quadráticos da RNA, IEEE-118.

\begin{tabular}{|c|c|c|c|}
\hline Caso & $\begin{array}{c}\text { Conjunto de } \\
\text { Treinamento }\end{array}$ & $\begin{array}{c}\text { Conjunto de } \\
\text { Validação }\end{array}$ & $\begin{array}{c}\text { Conjunto de } \\
\text { Teste }\end{array}$ \\
\hline Caso-2 & 0,0215 & 3,8819 & 3,5107 \\
\hline Caso-3 & 0,3357 & 3,8080 & 3,4548 \\
\hline
\end{tabular}

Para este sistema, o Caso-2 implica em maior aproximação e maior eficiência da RNA, ao contrário do ocorrido para o sistema IEEE-14. Isto demonstra que o maior número de informações para a RNA, como no Caso-3, não implica necessariamente em aumento de seu desempenho, uma vez que sua complexidade aumenta.

Destaca-se que os tempos de treinamento da RNA para os IEEE-14 e IEEE-18 são, respectivamente, 37,41 s e 67,83 s. Observa-se, neste caso, que estes tempos são reduzidos. No entanto, mesmo que sejam elevados para outros sistemas isto não inviabiliza a aplicabilidade do método, uma vez que o treinamento da RNA é offline. Ou seja, este tempo não deve ser computado na operação online e em tempo real da RNA. 
Contudo, mudanças topológicas no sistema requerem novo treinamento offline.

\section{CONCLUSÕES}

Este trabalho apresentou uma análise de aplicação de inteligência artificial, através da utilização de redes neurais artificiais, ao problema de avaliação de confiabilidade composta de sistemas elétricos de potência. A análise consistiu na avaliação de impactos no desempenho do cálculo de índices de confiabilidade devido à utilização de diferentes entradas da rede, como a geração total disponível e a geração disponível por barra. Conclui-se que a melhor opção não pode ser previamente definida, pois pode variar entre sistemas. Além disto, os resultados demonstram que a eficiência computacional aumenta significativamente, haja vista a redução do tempo de processamento com a utilização de RNAs. Este resultado demonstra que esta aplicação é promissora e que novas entradas devem ser investigadas, tais como a disponibilidade de linhas de transmissão, o que será incluído como proposta para futuros trabalhos. Adicionalmente, outra proposta será a incorporação de um fluxo de potência linearizado (DC), em uma etapa anterior ao fluxo de potência ótimo, visando identificar preliminarmente estados falhos e evitando a execução do fluxo de potência ótimo em casos de sucesso.

\section{AGRADECIMENTOS}

Os autores desse artigo agradecem o apoio da FAPEMIG, CAPES, CNPq, INERGE.

\section{REFERENNCIAS}

Allan, R., and Billinton, R. (2000). Probabilistic Assessment of Power Systems. Proceedings of the IEEE, vol. 88, no. 2, pp. 140-162.

Billinton R., and Li, W. (1993). A system state transition sampling method for composite system reliability evaluation. IEEE Transactions on Power Systems, vol. 8 no. 3, pp. 761-770.

Billinton, R., and Li, W. (1994). Reliability assessment of electric power system using Monte Carlo methods. New York: Plenum.

Billinton R., and Allan, R. (1996). Reliability Evaluation of Power Systems. Plenum Press New York and London, pp. 410.

Carvalho, M.F., Soares, S. e Ohishi, T. (1988). Optimal active power dispatch by network flow approach. IEEE Transactions on Power Systems vol. 3 no. 4.

Dias. J. A. S. (2014). Modelo para representação de séries temporais correlacionadas com aplicação na avaliação da confiabilidade por simulação Monte Carlo NãoSequencial. Universidade Federal do Rio de Janeiro, Rio de Janeiro, pp. 21.

Leite da Silva, A.M., Manso, L.A.F., Mello, J.C.O., and Billinton, R. (2000). Pseudo-chronological simulation for composite reability analysis with time varying loads. IEEE Trans. on Power Systems. vol. 15, no.1, pp.73-80.
Leite da Silva, A.M., Resende, L.C., Manso, L.A.F., and Miranda, V. (2007). Composite reability assessment based on Monte Carlo simulation and artificial neural networks. IEEE Trans. on Power Systems. vol. 22, no. 3, pp. 1202-2019.

Luo, X., Singh, C., and Patton, A. D. (2000). Power system reliability evaluation using self organizing map. Proc. Power Eng. Soc. Winter Meeting, Jan. 23-27, 2000, vol. 2, pp. 1103-1108.

Melo, A.C.G., Pereira, M.V.F. e Leite da Silva, A.M. (1992). Frequency and duration calculations in composite generation and transmission reliability evaluation. IEEE Trans. on PWRS, vol. 7, no. 2, pp. 469-476.

Mello, J. C. O., Pereira, M. V. F., and Leite da Silva, A. M. (1994). Evaluation of reliability worth in composite system based on pseudo-sequential Monte Carlo simulation. IEEE Trans. Power Syst., vol. 9, no. 3, pp. 1318-1326.

Mitra, J., and, Xu, X. (2010). Composite system reliability analysis using particle swarm optimization. IEEE 11th International Conference on Probabilistic Methods Applied to Power Systems, Singapore, 2010, pp. 548-552.

Oliveira, A. R. L., Filho, S. S. (2003) Métodos de Pontos Interiores para Problema de Fluxo de Potência Ótimo DC. Revista Controle \& Automação vol.14 no 3, pp. 278.

Pereira, M.V.F., and Balu, N.J. (1992). Composite generation/transmission reliability evaluation. Proceedings of the IEEE, vol. 80, no. 4, pp. 470491.

Resende, L. C., Manso, L. A. F., Dutra, W. D., and Leite da Silva, A.M. (2015). Support Vector Machine application in composite reliability assessment. 18th International Conference on Intelligent System Application to Power Systems (ISAP), Porto, 2015, pp. 1-6.

Rosa, M., Leite da Silva, A.M., and Miranda, V. (2012). Multiagent systems applied to reliability assessment of power systems. International Journal of Electrical Power \& Energy Systems, pp. 367-374.

Song, Y., Bu, G., and Zhang, R. (2005). A fast method probabilistic reliability assessment of bulk power systems using FSOM neural network as system states filters. Proc. IEEE/Power Eng. Soc. Transmission and Distribution Conf. Exhib.: Asia and Pacific, pp. 1-6.

Terra., L.D.B. (1989). A global Methodology for reactive power management and voltage control in power system. Imperial College, London, pp 189.

Urgun, D., and Singha, C. (2018). Multi label RBF classification method for composite system reliability evaluation. IEEE International Conference on Probabilistic Methods Applied to Power Systems (PMAPS), Boise, ID, 2018, pp. 1-5.

$\mathrm{Xu}, \mathrm{X}$., and Mitra, J. (2011). Reliability evaluation of composite system using bacterial foraging algorithm. 17th Power Systems Computation Conference.

Electrical and Computer Engineering (2019). www.ee.washington.edu/research/pstca. 